\title{
Construyendo una estrategia ambiental para la región metropolitana de San Salvadorl
}

\section{Introduccion}

Década de los noventa. En El Salvador acaba de finalizar una guerra que ha durado un poco más de diez años $y$, en medio de un dinámico proceso de construcción de un régimen político democrático y la reconstrucción social y material del país, emerge con singular fuerza una problemática de enorme gravedad que el conflicto político militar había postergado: el acentuado proceso de degradación ambiental que se había iniciado mucho tiempo atrás.

Pero el surgimiento a la luz pública, y la toma de conciencia ciudadana sobre la magnitud de este problema, se enfrenta a enormes vacíos: por un lado, la ausencia de estudios sobre las características y causas de este proceso de degradación debido al desmantelamiento de los centros académicos y unidades gubernamentales, que en los años sesenta y principios de los setenta habían comenzado su estudio; y por otro, el estancamiento en el desarrollo de la institucionalidad responsable de su gestión. A lo anterior se suma la poca organización ciudadana en tomo a esta problemática.

No obstante, esta situación tiende a cambiar rápidamente y comienza a organizarse la sociedad civil; llegan importantes aportes financieros y apoyo técnico de organismos e instituciones de ayuda internacional, y se inicia una aguda discusión sobre el marco legal e institucional de lo que debería regular el uso de los recursos y servicios ambientales y promover su desarrollo, que culminará con la creación del Ministerio del Medio Ambiente y Recursos Naturales y la aprobación de la Ley del Medio Ambiente en 1998.

El apoyo financiero y técnico antes mencionado conduce a la elaboración de numerosos diagnósticos planes sectoriales por parte de consultores extranjeros y 
nacionales. Orientados a la formulación de políticas, estos estudios y propuestas no pueden, sin embargo, suplir la necesidad de realizar estudios en profundidad sobre las causas de la problemática ambiental que vive el país. Por otra parte, por su carácter sectorial (hídrico, forestal, energético, etc.) no abordan con un enfoque integral los procesos ambientales. Entre los pocos estudios con esta visión se destaca un análisis que relaciona la dinámica ambiental con la evolución de la economía y la población (PRISMA, 1995).

Lo anterior se refleja en la escasa atención brindada a las ciudades, que condensan y vinculan los problemas que normalmente aparecen separados en las Llamadas agendas "verde" y "café". Esto es particulamente grave en el caso salvadoreño por la limitada extensión del país y las fuertes restricciones a que está sometido en términos de sus recursos y servicios ambientales.

En este contexto, este trabajo aborda la situación en la Región Metropolitana de San Salvador (RMSS), ámbito territorial donde se ubica la principal concentración urbana del país, el Area Metropolitana de San Salvador (AMSS) y casi un tercio de la población nacional, apoyándose en varias investigaciones realizadas en años anteriores, especialmente el estudio que llevó a cabo el centro de investigaciones PRISMA con apoyo de la Agencia Intemacional para el Desarrollo (AID), del gobierno de Estados Unidos, del cual retomamos muchos de los datos y algunas conclusiones. Obviamente, la responsabilidad del contenido de este trabajo es exclusiva de sus autores.

\section{El marco analítico}

La construcción de una estrategia ambiental para la Región Metropolitana de San Salvador (RMSS) que contribuya a impulsar el desarrollo sostenible del país, requiere relacionar la población que vive y utiliza este ámbito territorial con disponibilidad de los recursos y servicios ambientales y la contaminación de los mismos, lo que conduce a la cuestión de la capacidad de soporte del medio ambiente natural y construido.

Esta capacidad de soporte no debe entenderse como la población máxima que puede albergar este territorio, sino como la máxima carga que puede ser impuesta por la población al mismo sin provocar procesos de degradación irreversibles. Incluye, entonces, el consumo y la contaminación de los recursos y servicios ambientales, además del número de habitantes. Por ello, una estrategia ambiental de este tipo debe promover procesos que no transgredan los umbrales críticos y generen daños ambientales irreversibles.

Respetar estos umbrales críticos implica construir un marco de derechos y deberes ambientales por el carácter limitado de los bienes y servicios ambientales, y por constituir éstos bienes comunes. Las formas en cómo se perciben estos derechos y deberes, así como la disposición para asumir los costos económicos y 
sociales del agotamiento y la contaminación de los bienes y servicios ambientales, y su distribución entre los distintos grupos sociales, es un punto fundamental para establecer acuerdos sobre la cual se sentarán las bases para un desarrollo sostenible. Un ejemplo claro al respecto es el agua.

La visión expresada en las líneas anteriores requiere algunas precisiones sobre el proceso de urbanización y el desarrollo sostenible.

Hace diez años, el documento Our Commond Future (Brundıland y cols., 1987) planteó que el crecimiento urbano era uno de los fenómenos más importantes por considerar para impulsar el desamollo urbano sostenible ${ }^{2}$. Frente a la concentración de población en grandes ciudades, el incremento de la pobreza urbana, etc., la Comisión Brundtland recomendaba: formular estralegias urbanas a nivel nacional; reforzar los gobiemos locales a través de la descentralización; integrar los planes de desarrollo urbano y rurales, etc.

Hasta la fecha se han hecho innumerables críticas al planteamiento de la Comisión Brundıland, especialmente al hecho de que no aborda los límites de la capacidad de soporte de los recursos naturales. Para el caso de las ciudades podría agregarse la necesidad de lomar en consideración la capacidad de soporte del medio físico construido. Por su parte, el Fondo Mundial para la Naturaleza (WWF) sostiene que el desarrollo sostenible tiene por eje el mejoramiento de la calidad de vida humana, manteniéndose dentro de los límiles que impone la capacidad de carga de los ecosisternas (Stedman y Reed, 1996). Esta concepción" relaciona entre sí tres componentes básicos: el social, el ambiental y el económico. Criticando el absoluto predominio de las mediciones económicas del desarrollo, exige diferenciar el capital construido por el ser humano del capital humano y del capital natural. A esta última trilogía podría agregarse el capital social (Coleman, 1988). Retomando estas ideas, podríamos decir que:

En el ámbito de las ciudades, el desarrollo sostenible supone desarrollar el capital humano equitativamente, potenciando el desarrollo del capital social y el capital construido, mientras se preserva el capital natural.

A principios de los noventa, el Banco Mundial publicó su agenda urbana para esta década (Banco Mundial, 1991). El planteamiento del Banco Mundial ha recibido varias críticas, especialmente por su polémica noción de "productividad urbana" (Burgess y cols., 1997). Casi simultáneamente, el Programa de las Naciones Unidas para el Desarrollo (PNUD) lanzó su Programa de Cooperación para el Desarrollo Urbano (UNDP, 1991), donde proponía un enfoque multidimensional para enfrentar la problemálica del medio ambiente urbano mediante la estimulación de la cooperación entre los diferentes actores, públicos y privados; el mejoramiento de la regulación; el trabajo a nivel local y la promoción de la participación de las comunidades urbanas de base, y el desarrollo de estrategias a largo plazo. 
La virtud de estas posiciones consiste en que han ido impulsando, poco a poco, el desarrollo de metodologías para el análisis y la formulación de políticas de la problemática que nos ocupa. A continuación analizaremos algunos de los esfuerzos hechos en esta área.

En un trabajo reciente, uno de los funcionarios de alto nivel del Banco Mundial (Serageldin, 1995) trató de avanzar en la operacionalización del concepto de desarrollo sostenible. En un primer momento se critica la visión economicista prevaleciente en el Banco, la cual reduce la dimensión económica al simple crecimiento y la eficiencia, la dimensión ecológica al manejo de los recursos naturales, y la dimensión social a la reducción de la pobreza. Plantea, entonces, la necesidad de incorporar en la primera la cuestión de la equidad; en la segunda, la capacidad de soporte, la biodiversidad y otras cuestiones globales; y en la tercera dimensión, el empoderamiento, la participación, la movilidad, la cohesión social, la identidad cultural y el desarrollo instilucional.

A partir de esto, el Banco Mundial está tratando de desarrollar la concepción de la "sostenibilidad como oportunidad", la cual se basa en la diferenciación de los tipos de capital y la proporción en que una opción de desarrollo sostenible debe heredar éstos a las generaciones futuras. El cálculo del capital humano, y sobre todo del capital social, constituye un gran desafío que se empieza a enfrentar. Otro reciente trabajo del Banco Mundial sobre pobreza urbana constituye un buen ejemplo al incorporar criterios para medir la vulnerabilidad urbana y el capital social (Moser, 1996).

Respecto a las ciudades, se han realizado ya muchos trabajos sobre el medio ambiente urbano y su relación con la sostenibilidad del desarrollo. Uno de ellos explora las dimensiones de las sostenibilidad, y sugiere que el desarrollo urbano sostenible puede alcanzarse a través de la reestructuración de la economía y los sistemas sociales a niveles internacional, nacional, regional y local. Metodológicamente, lo interesante es la vía propuesta para dicha reestructuración: al identificar los "puntos de presión ambiental" en las ciudades, entre los cuales se identifican las oportunidades de trabajo, la pobreza, la salud, la oferta de alimentos, la vivienda, la energía, el agua polable, la tierra urbana, los desechos sólidos, etc. (Stren, While y Whilney,1992).

Desde la perspectiva de los organismos de cooperación internacional, la propuesta más elaborada es la del Programa de Gestión Urbana (PGU) ${ }^{4}$ (Bartone y cols., 1994). El punto de partida en su planteamiento es la identificación de la llamada "agenda café" (Brown Agenda), constituida por un conjunto de problemas que inciden en la pobreza, la salud y la productividad urbanas, y que acompañan a los problemas de la "agenda verde" (Green Agenda): la disminución de los recursos acuíferos, energéticos, forestales, la degradación de la tierra, la ocupación de zonas de riesgo, etc. Además, señala cinco factores que determinan la variabilidad de los problemas ambientales urbanos: $(a)$ las características únicas 
de cada área urbana; (b) el tamaño de la población y las tasas de crecimiento; (c) el nivel de ingresos y el desarrollo económico; (d) las distintas dimensiones espaciales de los problemas ambientales urbanos; y $(e)$ el rol de los actores locales.

Ante estos factores, una estralegia ambiental urbana debe enfrentar tres fuentes de tensión que generen falsas dicotomías: los enfoques integrales frente a los sectoriales; los análisis de situación versus los análisis de proceso; y los enfoques descentralizados frente a los centralizados. Su solución permitirá manejar "trade-offs" económicos y políticos como las economías de escala y el incremento de los costos ambientales; la asignación de subsidios; el paso de una política "verde" hacia una política de "justicia ambiental"; las limitaciones presupuestarias, etc.

En este trabajo partimos de que el desarrollo urbano está determinado por cuatro elementos: la población, el soporte fisico, las actividades que se realizan y la gestión (Lungo et al, 1997). Las relaciones entre estos cuatro elementos permiten identificar varios aspectos de esta problemática, entre ellos, la explotación de los recursos, la concentración poblacional, la regulación y la participación urbana, etc. De estos elementos, el lugar primordial lo ocupa la población, sujeto central del desarrollo sostenible. Las relaciones entre ellos no tienen un carácter unidireccional y vinculan a los distintos componentes mediante la formación de una trama compleja y cambiante.

\section{Gréfice 1 \\ Desarrollo urbano y sostenibilidad}

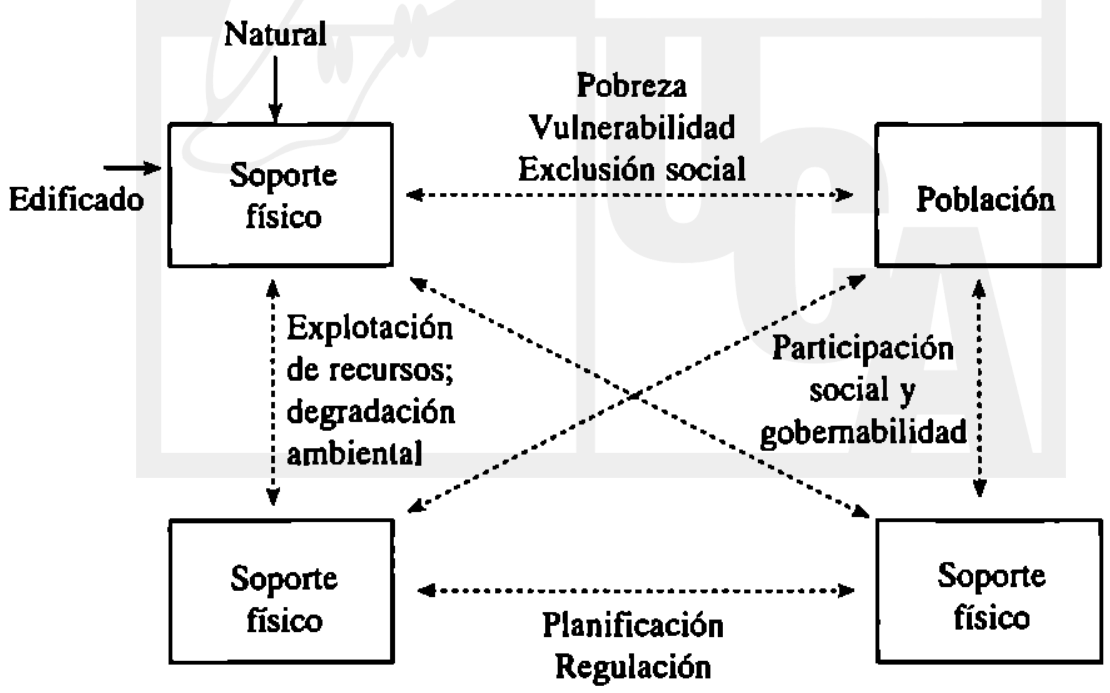


Al considerarse a la población como el factor central del desarrollo, las formas de gestión territorial son claves para impulsar una estrategia ambiental que contribuya al desarrollo sostenible al minimizar los coslos sociales y ambientales. Los procesos descritos anteriormente se circunscriben alrededor de tres parámetros fundamentales: las condiciones de vida de la población, la productividad de la economía urbana y la gobernabilidad urbana.

En el caso que nos ocupa, la RMSS, el acelerado crecimiento poblacional, la dinámica de sus economía, los altos niveles de pobreza y exclusión social, y la forma irracional en la ocupación de su territorio se explican por la forma de gestión actual de este ámbito territorial, caracterizada por las conlradicciones, debilidades y alto grado de obsolescencia de los marcos institucional y regulatorio responsables del desarrollo urbano.

\section{Los principales problemas del medio ambiente en la Región Metropoli- tana de San Salvador}

En un trabajo anterior (Lungo et al, 1997) hemos delimitado la RMSS con base en criterios territoriales, ambientales y económicos. Comprende un área de $1,020 \mathrm{Kms}^{2}$ y casi 2 millones de habitantes. Incluye a 24 municipios casi en su totalidad, entre ellos los catorce que conforman el Area Metropolitana de San Salvador. Entre las principales características que inciden en la degradación ambiental en este ámbito territorial podemos señalar las siguientes: (a) concentra un tercio de la población y la mayor parte de las actividades económicas más dinámicas del país. (b) Este crecimiento económico y poblacional conduce a un aumento de la demanda y consumo de bienes y servicios ambientales, sin contar con la adecuada capacidad de preservación y desarrollo de los mismos ni con la posibilidad de absorción de los desechos y control de los procesos contaminantes. (c) Los procesos de degradación ambiental se caracterizan, en su mayoría, por ser antrópicos, y son producto de la forma en que se desarrollan la economía y los distintos procesos de la reproducción social y la obsolescencia y debilidad de la gestión territorial y ambientals, lo cual genera impactos negativos que tienden a sobrepasar los umbrales críticos.

Efectivamente, en la RMSS es donde se observan las tasas más altas de crecimiento poblacional del país, que generan una concentración de población tal que, entre 1950 y 1992, su proporción con respecto al país ha pasado de 19.94 a 33.38 por ciento, respeclivamente, debido, entre otras causas, a la ubicación en ella de la mayoría de las actividades industriales en los años sesenta, los procesos de migración interna a causa del conflicto armado en los ochenta y la crisis del sector agropecuario en esta década. En su interior son visibles tendencias diferenciadas. Se destaca el municipio central de San Salvador por la disminución de su importancia demográfica dentro del Area Metropolitana de San Salvador (AMSS), donde pasa de concentrar el $\mathbf{8 0}$ por ciento de su población en 


\section{Gráfica 2 \\ La región Metropolitana de San Salvador}

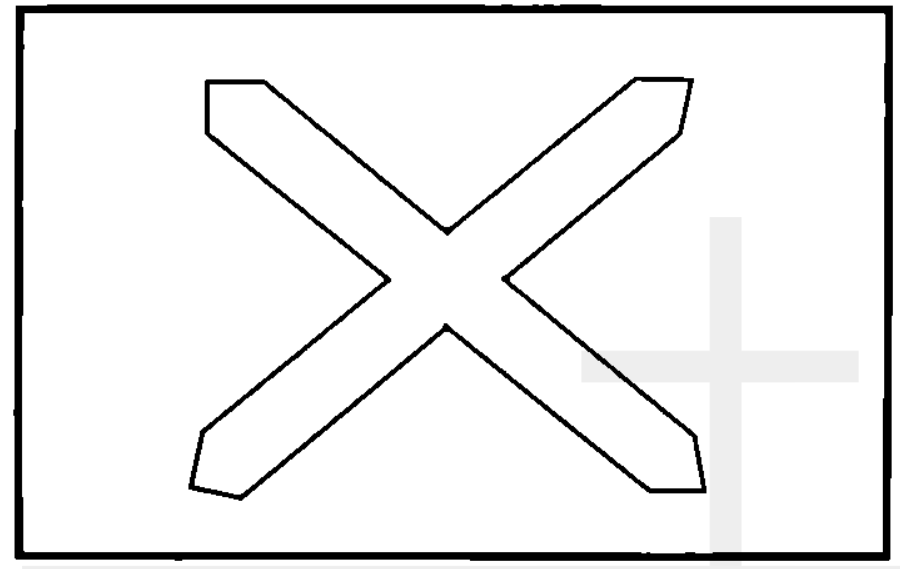

1950 , a 40 por ciento en 1992, aun y a pesar de que su densidad poblacional se triplicó durante este período. Otra tendencia importante por sus consecuencias ambientales es la disminución del ritmo de crecimiento de los municipios del este, mientras aumenta en el norte y el centro, y sobre todo en los sectores oeste y suroeste, lo cual incrementa la presión territorial en estas áreas.

Económicamente, la RMSS concentra el 53 por ciento del Producto Interno Bruto del país, de donde se destacan los sectores de la construcción y el transporte. El sector industrial sigue siendo importante, aunque ha modificado su estructura interna y ha dado paso al desarrollo del sector de las maquilas (Cuervo et al, 1998). Un proceso importante por destacar es la terciarización de la economía metropolitana. Entre las características de este sector, además de su tamaño, está el fuerte carácler informal del comercio y los servicios. Geográficamente se nota una mayor presencia de la inversión comercial y financiera en el municipio central de San Salvador, que lo convierten en una zona orientada al consumo, mientras las áreas residenciales se concentran en la periferia del AMSS. Dichas modificaciones en la estructura espacial están relacionadas con los problemas del tráfico que se experimentan en la actualidad.

Aunque a nivel nacional el sector industrial tampoco ha disminuido su importancia, éste se ha volcado también hacia la maquila, lo que se expresa en la mayor inversión y niveles de exportación experimentados dentro de este rubro ${ }^{6}$. Cabe mencionar que dentro de la RMSS se encuentra localizada la mayor producción de productos no tradicionales del sector industrial y maquila, que concentra el 79.25 por ciento del total de empresas del primero y el 81.48 por ciento de la segunda. Esto tiene consecuencias ambientales importantes por la movilización del personal y de las mercancías que genera y la obsolescencia del siste- 
ma vial existente, que se suma a los altos niveles de contaminación que generan algunos procesos productivos industriales (Cuervo et al, 1998).

A nivel territorial, los datos del recientemente elaborado Plan Maestro de Desarrollo Urbano para el Area Metropolitana Ampliada-PLAMADUR (VMVDU, 1997) muestran que durante los últimos treinta años, el área urbanizada del AMSS casi ha duplicado su extensión, pasando de 5,200 hectáreas en 1966 a casi 10,000 en 1995; esta ocupación es extensiva, discontinua y desigual en términos de dotación de servicios y equipamientos. Las hipótesis formuladas por el PLAMADUR, que pretenden un crecimiento programado para reducir la actual concentración demográfica y económica, han estimado que entre 1995 y el año 2005 se necesitarían 3,315 hectáreas adicionales, de las cuales 1,530 se destinarían al uso habitacional.

Durante los últimos años ha habido un crecimiento de urbanizaciones que se han ubicado en áreas ecológicamente frágiles y una tendencia a urbanizar tierras agrícolas en la periferia del AMSS, especialmente al norte y nororiente, las cuales impactarán negativamente en los recursos hídricos dada la existencia de amplias zonas de alta infiltración; esto se suma al problema del manejo de los desechos sólidos, la contaminación industrial por desechos, y la del aire por el prevesible incremento de los flujos de transporte.

En términos de la gestión del desarrollo urbano, el panorama existente muestra una estructura institucional dispersa y contradictoria, con múltiples instancias que poseen dependencias relacionadas con el medio ambiente y la gestión del suelo urbano, sin contar con una política integral ni con instrumentos de acción adecuados ni la existencia de una entidad coordinadora que posibilite la unificación de las decisiones. En este sentido, la acción del Estado obedece más a dinámicas coyunturales que a una política clara de gestión territorial y ambiental. Ante los conflictos se privilegian las sanciones legales por la ausencia de mecanismos que permitan la negociación y resolución de los mismos (Gómez, 1998).

Es urgente, entonces, la construcción de una estrategia ambiental que reconozca la importancia de los impactos del desarrollo urbano sobre el medio ambiente, que permita la participación ciudadana y promueva la creación de instrumentos y espacios para la resolución de los conflictos provenientes de este desarrollo.

Para una mejor comprensión de los distintos procesos de la degradación ambiental y contribuir a la construcción de una estrategia ambiental en la RMSS, a continuación se presenta información puntual sobre la ocupación de zonas de alto riesgo, el aprovisionamiento de agua y su contaminación por vertidos industriales, la generación y manejo de los desechos sólidos, y la contaminación del aire.

La ocupación de zonas de riesgo dentro de la RMSS se debe a la ausencia de una adecuada gestión del suelo, lo que genera riesgos a desastres, dada la combi- 
nación de amenazas naturales y antrópicas, y la vulnerabilidad socioeconómica imperante. Otro fenómeno que muestra la inadecuada gestión del suelo es la alteración irracional de la topografía (nivelación de terrenos con altas pendientes, relleno de quebradas, etc.) que altera el curso natural de las aguas superficiales lo cual acentúa la sismicidad que caracteriza a esta zona (Boomer et al, 1998).

Respecto a la provisión y contaminación de agua, el carácler extensivo del proceso de urbanización reduce las capacidades de infiltración debido a que la ciudad se ubica en una zona geológica altamente permeable, disminuyendo así la capacidad de absorción de los mantos acuíferos. La insuficiencia en la producción se ve agrabada por los serios problemas de contaminación de este recurso. Recientes estudios muestran (UCA/FLAES, 1997) que las aguas superficiales del país se encuentran contaminadas en un $\mathbf{9 0}$ por ciento, problema que se acentúa dado que éstas constituyen dos tercios de los recursos hídricos potencialmente disponibles. La contaminación es generada por desechos orgánicos, agroquímicos e industriạes, pero los mayores problemas de contaminación se deben a los desechos industriales. En algunos ríos la contaminación es tal, que no se debería permitir su consumo para uso doméstico.

Sobre la contaminación industrial por vertidos, datos del Ministerio de Salud Pública y Asistencia Social (MSPYAS, 1995) mostraban que de un total de 120 industrias que operaban dentro del AMSS, únicamente 16 contaban con algún sistema de tratamiento de aguas residuales, y aunque ha habido una mejora entre la situación encontrada en 1994 y en 1997, pasando de 4 a 12.7 por ciento las industrias que acceden a alcantarillado con tratamiento previo, la situación es grave en términos de reducción y eliminación de esta fuente de contaminación.

La problemática de los desechos sólidos se ha tornado en un punto polémico de la agenda nacional, y constituye la expresión latente de las extemalidades negativas generadas por el actual modelo de desarrollo. Sobre la misma podemos mencionar que (García, 1997): (a) la industria nacional presenta procesos de producción que generan bienes no preparados para reingresar a la producción vía la reutilización y/o reciclaje, y tanto la legislación existente como las características propias del sector presentan barreras para que propicien cambios en estos procesos. (b) Desde 1989 ha habido un fuerte proceso de eliminación de barreras arancelarias y no arancelarias que ha incrementado el consumo de productos importados, los cuales tampoco cuentan con sistemas de reincorporación de los desechos al flujo de producción. (c) Hasta el momento no se cuenta con iniciativas que promuevan acciones para la disminución de la producción de desechos. Por lo anterior, en el país solamente una pequeña parte de la producción se recicla. (d) En el AMSS se estima que el 50 por ciento de la basura no se recolecta; dicho servicio es brindado por las municipalidades $y$ enfrenta problemas presupuestarios, lécnicos y de personal adecuado para llevar a cabo dicha labor. 
La contaminación del aire incide fuertemente en los niveles de morbilidad y, por tanto, las enfermedades respiratorias son las causantes de más del $\mathbf{4 0}$ por ciento de las muertes infantiles (Orellana, 1997). En el ámbito metropolitano, esta problemática está asociada con el crecimiento del parque vehicular, así como con la antigüedad y las pésimas condiciones de mantenimiento del mismo. Esto se agrava por la obsolescencia de la infraestructura vial (MOP, 1997), así como por el patrón actual del uso de suelo.

\section{Cuadro 1}

RMSS: escenario ambiental socialmente no sostenible

\begin{tabular}{|c|c|c|}
\hline Procesos estructurantes & Riesgos & Umbrales crificos \\
\hline - Crecimiento poblacional & $\begin{array}{l}\text { Disminución de la } \\
\text { calidad de vida }\end{array}$ & $\begin{array}{l}\text { Población mayor a la } \\
\text { capacidad de carga }\end{array}$ \\
\hline - Dinámica económica & $\begin{array}{l}\text { Disminución de la } \\
\text { productividad urbana }\end{array}$ & $\begin{array}{l}\text { Conlinuidad del } \\
\text { modelo aclual }\end{array}$ \\
\hline $\begin{array}{l}\text { Procesos de degradación } \\
\text { ambiental }\end{array}$ & Riesgos & Umbrales críricos \\
\hline - Uso del suelo & $\begin{array}{l}\text { Ocupación de zonas frágiles } \\
\text { y de riesgo }\end{array}$ & $\begin{array}{l}\text { Superación de cota límite en } \\
\text { el volcán San Salvador } \\
\text { y el cerro San Jacinto; la } \\
\text { deforeslación de la cordillera } \\
\text { del Bálsamo más allá del nivel } \\
\text { actual; la no erradicación de } \\
\text { los asentamientos en riesgo; la } \\
\text { urbanización no controlada de } \\
\text { Zaragoza, Zapotitán, Tonacate- } \\
\text { peque y Apopa. }\end{array}$ \\
\hline - Recursos hídricos & $\begin{array}{l}\text { Reducción de la superficie } \\
\text { de infiltración para la recarga } \\
\text { de aguas sublertáneas y } \\
\text { contaminación de las fuentes } \\
\text { de agua }\end{array}$ & $\begin{array}{l}\text { Déficit de aprovisionamiento } \\
\text { mayor al } 50 \% \text { e incremento de } \\
\text { enfermedades gastrointestinales } \\
\text { a más del } 35 \%\end{array}$ \\
\hline - Desechos s6́lidos & $\begin{array}{l}\text { Incremento en generación y } \\
\text { disminución de capacidad de } \\
\text { manejo }\end{array}$ & $\begin{array}{l}\text { Generación más de } 1,500 \\
\text { toneladas y reducción de la } \\
\text { recolección a menos del } 50 \%\end{array}$ \\
\hline
\end{tabular}


- Contaminación industrial

- Contaminación del aire
Contaminación por desechos en nuevas zonas $\mathrm{e}$ incremenlo de las actuales

Contaminación por aumento y falta de control de vehículos; aumento de viajes y dislancias de recorridos
Porcentaje de industrias sin Iratamiento mayor del 50\%

Aumenlo del $20 \%$ de la lasa de motorización actual; control de vehículos a menos del $80 \%$; incremento del número de viajes y recorridos; más del $40 \%$ de enfermedades respiratorias.

\begin{tabular}{lll}
\hline Gestión ambiental & Riesgos & Umbrales críticos \\
\hline $\begin{array}{l}\text { * Ausencia de políticas } \\
\begin{array}{l}\text { * Fragmentación } \\
\text { institucional }\end{array}\end{array}$ & $\begin{array}{l}\text { Agravamiento de } \\
\text { la degradación }\end{array}$ & $\begin{array}{l}\text { Nivel de costos socialmente } \\
\text { sostenibles }\end{array}$ \\
$\begin{array}{l}\text { Falta de regulaciones } \\
\text { e instrumentos económicos de conflictos }\end{array}$ & $\begin{array}{l}\text { Existencia de siluaciones de } \\
\text { ingobemabilidad }\end{array}$ \\
\hline
\end{tabular}

El panorama antes descrito se suma a los altos niveles de pobreza y exclusión social, y los incipientes procesos de participación social; estas situaciones constituyen serios obstáculos que deben ser superados para polenciar el capital social y construir nuevas relaciones de gobernabilidad urbana (Lungo, 1998), modificar radicalmente el actual marco regulatorio y la institucionalidad responsable del desartollo de la RMSS, además de introducir criterios ambientales en la edificación del marco construido y el manejo de los recursos y servicios ambientales con que cuenta.

La importancia de la gestión territorial como elemento clave para impulsar una estrategia ambiental aparece aqui en toda su importancia, por lo que es necesario ir más allá del análisis de los procesos de degradación en curso. Es necesaria la identificación de los principales actores involucrados, de sus percepciones e intereses, para poder impulsar modalidades de gestión basadas en la construcción de consensos.

\section{Los actores y sus posiciones}

La degradación ambiental en la RMSS se ha convertido, en los años recientes, en un punto de debate en el país, y se ha iniciado la búsqueda de medidas 
que protejan los recursos, mitiguen la contaminación y ordenen de manera sostenible el desarrollo de este ámbito territorial' ${ }^{7}$.

Pero la elaboración de una estrategia de desarrollo urbano sostenible debe basarse en el reconocimiento de la existencia de una serie de actores que están inmersos dentro de distintos espacios, niveles de poder y que tienen distintas percepciones e intereses ambientales. Para avanzar en su conocimiento hemos identificado las distintas formas asociativas existentes, lo que permite la construcción del mapa de relaciones internas y visualizar los puntos de convergencia y divergencia más relevantes.

En un primer momento, los actores pueden ubicarse en dos planos fundamentales: el del Estado y el de la Sociedad Civil. Obviamente esta simplificación de los aclores inmersos dentro de la gestión urbana no puede dar cuenta de la complejidad y heterogeneidad existente. Hay autores que señalan la existencia de sectores sociales expresados en el mercado, esfera que no sólo expresa el ámbito económico, aunque éste sea el más observable (Gómez, 1998).

Las diversas esferas de actuación social se producen dentro de un modelo de crecimiento económico, posibilitando identificar las distintas lógicas que se entrecruzan en el momento de definir políticas de gestión urbana: (a) la lógica estatal, donde se emanan las regulaciones para la realización de actividades y la resolución de conflictos. (b) La lógica del mercado, referida a los mecanismos de producción y distribución de bienes y servicios inmersos en el modelo económico que la sustenta. (c) La lógica de lo social, bajo lo cual confluyen distintos intereses privados que van desde la familia, las asociaciones voluntarias, asociaciones no gubernamentales, corporaciones y movimientos sociales, entre olros.

El reconocimiento de estas lógicas posibilita un mayor acercamiento al complejo panorama de diversos intereses que subyacen a las formas de distribución, regulación y/o consumo de los recursos y servicios ambientales en donde las formas de interacción asumen diferentes prioridades. Ante esta complejidad de intereses, muchas veces antagónicos, se precisa la construcción de una estrategia ambiental que contribuya al desarrollo sostenible.

También existe otro actor que se entrecruza con las lógicas anteriores; nos referimos a la cooperación internacional, cuyo rol ha generado impactos y orientaciones ambientales por medio del financiamiento de proyectos concretos.

En la RMSS, en tomo a la problemática ambiental, podemos plantear que se han constituido a plenitud dos actores: (a) las organizaciones ambientalistas (ONG's fundamentalmente), en reacción ante los agudos procesos de degradación del medio ambiente y con un sesgo predominantemente conservacionista; y (b) el gobierno central y algunos gobiernos locales, que reaccionan a su vez ante las demandas de las organizaciones ambientalistas. En menor medida aparecen configurándose como aclores algunas comunidades urbanas y los gremios empresariales, especialmente los constructores e industriales. 
Aunque la conciencia ciudadana se ha incrementado sustancialmente durante los últimos años, no se ha conformado un verdadero movimiento social ambiental en el país, lo que constituye una dificultad para la construcción de una estrategia ambiental para la RMSS. Las instituciones públicas, en medio de sus dispersas y contradictorias funciones, siguen siendo el actor mayoritario. El cuadro siguiente ejemplifica la complejidad existente de sus campos de acción en el interior del gobiemo central municipal.

\section{Cuadro 2}

Principales entidades del gobierno central y municipal con procesos ambientales dentro de la RMSS

\begin{tabular}{|c|c|c|c|c|c|}
\hline Entided & Procesas & Jerazquía & Unidad & Mandalo & Alcance \\
\hline $\begin{array}{l}\text { Minislerio del } \\
\text { Medio Ambiente } \\
\text { y Remursos } \\
\text { Naturales }\end{array}$ & $\begin{array}{l}\text { Todos los } \\
\text { procesos ambien- } \\
\text { lales }\end{array}$ & & $\begin{array}{l}\text { Exisien } \\
\text { diversas } \\
\text { unidades }\end{array}$ & $\begin{array}{l}\text { Desarrollo } \\
\text { ambiental en } \\
\text { forma general }\end{array}$ & \\
\hline $\begin{array}{l}\text { Ministerio de } \\
\text { Salud Pública } \\
\text { y Asisıencia } \\
\text { Social }\end{array}$ & $\begin{array}{l}\text { Conisminación } \\
\text { del agua y airc, } \\
\text { desechos sollidos } \\
\text { e industriales }\end{array}$ & Ministerial & $\begin{array}{l}\text { División de } \\
\text { saneamiento } \\
\text { ambiental }\end{array}$ & $\begin{array}{l}\text { Autorización, } \\
\text { sancamiento, } \\
\text { inspección, } \\
\text { conırol, } \\
\text { investigeción, } \\
\text { sanción }\end{array}$ & \\
\hline $\begin{array}{l}\text { Minisierio de } \\
\text { Trabajo }\end{array}$ & $\begin{array}{l}\text { Riesgos } \\
\text { industriales }\end{array}$ & & $\begin{array}{l}\text { Departamento } \\
\text { de higiene y } \\
\text { seguridad } \\
\text { ocupacional }\end{array}$ & $\begin{array}{l}\text { Autorización, } \\
\text { adminisıración, } \\
\text { conservación, } \\
\text { sanción }\end{array}$ & Nacional \\
\hline $\begin{array}{l}\text { Ministerio de } \\
\text { Agricultura y } \\
\text { Ganadería }\end{array}$ & $\begin{array}{l}\text { Recursos hidricos } \\
\text { y recursos } \\
\text { forestales }\end{array}$ & & $\begin{array}{l}\text { Dirección } \\
\text { general de } \\
\text { recursos } \\
\text { naturales } \\
\text { renovables }\end{array}$ & $\begin{array}{l}\text { Aulorización, } \\
\text { administración, } \\
\text { conservación, } \\
\text { sanción }\end{array}$ & \\
\hline $\begin{array}{l}\text { Ministerio del } \\
\text { Interior }\end{array}$ & $\begin{array}{l}\text { Riesgos nalurales } \\
\text { y antropicos }\end{array}$ & & $\begin{array}{l}\text { Comite de } \\
\text { emergencia } \\
\text { nacional }\end{array}$ & $\begin{array}{l}\text { Identificación de } \\
\text { zonas de riesgo, } \\
\text { intervención en } \\
\text { emergencies }\end{array}$ & \\
\hline $\begin{array}{l}\text { Ministerio de } \\
\text { Obras Públicas }\end{array}$ & Uso de suelo & & $\begin{array}{l}\text { Viceminisierlo } \\
\text { de vivienda y } \\
\text { desarrollo } \\
\text { usbano }\end{array}$ & $\begin{array}{l}\text { Planificacion, } \\
\text { reglamentación, } \\
\text { formulación y } \\
\text { ejecución de } \\
\text { polfícas }\end{array}$ & \\
\hline
\end{tabular}




\section{Cuadro 2 (Continuación)}

\begin{tabular}{|c|c|c|c|c|c|}
\hline Entidad & Procesos & Jerarquía & Unidad & Mandato & Alcance \\
\hline & $\begin{array}{l}\text { Control de conta- } \\
\text { minación del aire }\end{array}$ & Ministerial & $\begin{array}{l}\text { Viceministerio } \\
\text { de Transporte }\end{array}$ & $\begin{array}{l}\text { Control de } \\
\text { emisiones } \\
\text { y nuido }\end{array}$ & Nacional \\
\hline \multirow[t]{2}{*}{ Municipios } & Uso de surelo & Metropolitena & $\begin{array}{l}\text { Oficina de } \\
\text { Planificación del } \\
\text { AMSS }\end{array}$ & $\begin{array}{l}\text { Autorización, } \\
\text { planificeción, } \\
\text { ordenamiento, } \\
\text { protección, } \\
\text { sanción }\end{array}$ & Metropolitang \\
\hline & Desechos soblidas & Municipal & $\begin{array}{l}\text { Gerencia de } \\
\text { sancamiento } \\
\text { ambiental }\end{array}$ & $\begin{array}{l}\text { Sanesmiento, } \\
\text { recolección y } \\
\text { disposición final }\end{array}$ & Municipal \\
\hline $\begin{array}{l}\text { Asociación } \\
\text { Nacional de } \\
\text { Acueductos y } \\
\text { Alcantarillados }\end{array}$ & Agua & Aulónome & $\begin{array}{l}\text { Unidad de Medio } \\
\text { Ambienle }\end{array}$ & $\begin{array}{l}\text { Producción, } \\
\text { operación, } \\
\text { regulación, } \\
\text { abastecimiento }\end{array}$ & \multirow{5}{*}{ Nacional } \\
\hline $\begin{array}{l}\text { Asamblea } \\
\text { Legislativa }\end{array}$ & $\begin{array}{l}\text { Recursins } \\
\text { forestales, } \\
\text { recursos hidricos, } \\
\text { desectios solidos e } \\
\text { industriales, elc. }\end{array}$ & Legislaliva & $\begin{array}{l}\text { Comisión de } \\
\text { Salud y Medio } \\
\text { Ambiente }\end{array}$ & $\begin{array}{l}\text { Legislación, } \\
\text { mediación de } \\
\text { conflicios, } \\
\text { fiscalización, } \\
\text { invesligación }\end{array}$ & \\
\hline $\begin{array}{l}\text { Policía } \\
\text { Nacional Civil }\end{array}$ & $\begin{array}{l}\text { Recursos hidricos, } \\
\text { contaminación de } \\
\text { recursos } \\
\text { forestales, elc. }\end{array}$ & Ejecutivo & $\begin{array}{l}\text { Division del } \\
\text { medio ambiente }\end{array}$ & $\begin{array}{l}\text { Vigilancia, } \\
\text { inspección, } \\
\text { investigación, } \\
\text { sanción }\end{array}$ & \\
\hline $\begin{array}{l}\text { Fiscalla General } \\
\text { de la Repúblice }\end{array}$ & $\begin{array}{l}\text { Recursos } \\
\text { forestales, } \\
\text { recursos hidricos, } \\
\text { contaminacior, etc. }\end{array}$ & Judicial & $\begin{array}{l}\text { Unidad para la } \\
\text { defensa del } \\
\text { medio ambiente } \\
\text { y salud }\end{array}$ & $\begin{array}{l}\text { Investigación, } \\
\text { acusación, } \\
\text { punición }\end{array}$ & \\
\hline $\begin{array}{l}\text { Procuradurfa } \\
\text { pare la Defense } \\
\text { de los Derechos } \\
\text { Humanos }\end{array}$ & $\begin{array}{l}\text { Recursos } \\
\text { foresiales, } \\
\text { recursos hidricos, } \\
\text { contaminación, etc. }\end{array}$ & Autónoms & $\begin{array}{l}\text { Procuradurfa } \\
\text { Adjunta para el } \\
\text { Medio Ambienle }\end{array}$ & $\begin{array}{l}\text { Prolección, } \\
\text { investigación, } \\
\text { Iulela }\end{array}$ & \\
\hline
\end{tabular}

Fuente: a partir de Gómez, artículo citado. 
Durante la última década ha existido un proceso y un esfuerzo de modemización de la institucionalidad encargada de la gestión ambiental, esto no se ha logrado aún, a pesar de la creación en 1998 del Ministerio del Medio Ambiente y Recursos Naturales, y prevalece aún una estructura dispersa y descoordinada con múltiples instituciones que no cuentan con políticas integrales ni con instrumentos de acción conjunta, que tienen como resultado una fuerte debilidad institucional, multiplicación de intervenciones, contradicción de decisiones, elc., lo cual da paso a la desconfianza e incredulidad de estas acciones por parte de la ciudadanía.

El problema de este modelo no radica solamente en la aplicación sectorial y dispersa de las regulaciones ambientales, sino en la inexistencia de un ente coordinador del desarrollo y del medio ambiente con poder que posibilite un tratamiento global de los procesos. En ese sentido, el Estado sigue actuando a partir de intervenciones generadas por las demandas coyunturales.

La revisión de los principales conflictos dentro de la RMSS durante los últimos dos años revela dinámicas de conflictividad respecto a los procedimientos, forma de manejo de los recursos, decisiones y eficiencia de la gestión ambiental. El hecho más conflictivo hasta ahora registrado es el referido a la mala gestión de la tierra, que atenta contra la preservación de amplias zonas de recarga de los acuíferos subterráneos y de nacimientos de las aguas superficiales, mediante el crecimiento de los procesos de parcelación y urbanización que en muchos casos cuentan con el aval municipal.

Ante esta siluación poco alentadora, las organizaciones sociales deben reorientar su trabajo mediante el incremento de su vinculación con los gobiernos locales, pero manteniendo, en el caso de RMSS, una visión metropolitana que evile la parcialización de la visión de esta problemática y la atomización de las acciones de desarrollo ambiental.

\section{1. ¿Participación popular o participación social?}

Alrededor de la problemática ambiental urbana, como hemos indicado antes, interactúan muchos actores y se tejen innumerables y contradictorias relaciones, basadas en el hecho de que las percepciones sobre la relación entre el ser humano y la naturaleza son históricas y diferentes en cada cultura y para los distintos grupos sociales (Lungo, 1989). Efectivamente, cada sistema económico y social tiene una forma específica de explotación de los recursos naturales así como de utilización de la fuerza de trabajo humana $y$, en consecuencia, determinadas normas específicas sobre el "buen" y "mal" uso de ambos (Godelier, 1984).

Dada la diversidad de actores presentes, consideramos sumamente restrictivo hablar solamente de participación popular en la construcción de una estrategia ambiental para la Región Metropolitana de San Salvador. Para captar todas las relaciones y su complejidad, la estrategia debe incorporar a todos los actores que 
inciden en el medio ambiente en este ámbito territorial. Por eso sostenemos que debe hablarse de participación social o no restrictivamente de participación social.

¿Qué se entiende por participación social en la construcción de esta estrategia ambiental? Se trata de la participación de cada uno de los grupos sociales activos en este ámbito territorial que, al conservar sus particulares percepciones y aspiraciones sobre la relación entre el ser humano y la naturaleza, en un proceso de discusión con olros grupos sociales que tienen casa uno sus propios intereses, contribuyan a acercar puntos de vista diferentes, hacer concesiones y establecer consensos a partir de la aceptación de que los bienes y servicios ambientales comunes, limitados y no reproducibles permanentemente a volunlad.

¿Quién debería promover esta participación social? Pensamos, sin duda, que este rol le corresponde al Estado, en una articulación, en el caso de la RMSS, del gobiemo central y del conjunto de gobiernos municipales involucrados en este territorio. Obviamente lo anterior constituye una función inédita, no realizada antes por el Estado salvadoreño. Sin embargo, por tratarse de bienes y servicios comunes, de propiedad y derecho de uso del conjunto de la sociedad, y por su carácter perecedero, es el Estado quien debe promover el proceso de participación social tendiente a construir una estrategia ambiental para la RMSS. También es claro que por ser nueva esta función, la sociedad civil, a través de distintas instituciones, debe jugar un papel de "desencadenante" de estos procesos pero que reconozca y exija que el Estado cumpla esta función fundamental.

Para impulsarla, para poder ir edificando la nueva institucionalidad ambiental que la construcción de una estralegia ambiental requiere, es indispensable proponer espacios de discusión y concertación, e instrumentos de ejecución, innovadores, participativos y efectivos.

Una amplia participación social en la solución de los problemas ambientales de la RMSS es, además, una forma de construcción de ciudadanía social "desde abajo" (Jelin, 1997), lo que contribuiría a la implementación de la estrategia ambiental que se diseña.

\section{Contruyendo una estrategia ambiental para la Región Metropolitana de San Salvador (RMSS)}

En páginas anteriores hemos planteado la existencia de dos procesos estructurales claves que inciden en la degradación ambiental de la RMSS: el crecimiento poblacional y la dinámica de la economía. Aunque la estralegia ambiental tiene que referirse a procesos y problemas específicos, debe abordar estos procesos estructurales que constiluyen el contexto de los últimos.

Respecto al crecimiento poblacional, se podría sugerir la creación de estímulos para disminuir la migración interna hacia la RMSS y promover el desarrollo de otras regiones del país. La factibilidad de estas medidas depende del segundo 
proceso, particularmente por la poca importancia asignada al sector agropecuario en el actual modelo de crecimiento económico. Esto podría parecer contradictorio con uno de los puntos del marco analítico adoptado, que sostiene que el crecimiento poblacional no constituye un problema ambiental en sí mismo. Debemos recordar, no obstante, que agregábamos que la fuente principal de los procesos de degradación ambiental es la forma en que la población ocupa y utiliza el territorio. Respecto a la dinámica de la economía, que obedece también a los lineamientos del modelo de crecimiento económico, la estrategia ambiental debe, ante todo, impulsar acciones que intenten incidir en los procesos productivos anticipadamente, y no limitarse a medidas posteriores como la exigencia de la presentación de estudios de impacto ambiental para proyectos o procesos específicos ya definidos.

Es necesario, entonces, articular los programas y acciones parciales para que los beneficios obtenidos no se vean revertidos por efectos no previstos. Por ejemplo, los beneficios de la introducción de tecnologías limpias en la producción industrial pueden ser contrarrestados si no se establecen criterios ambientales para la ubicación de nuevos o la reubicación de antiguos establecimientos industriales. Lo mismo ocurre con la contaminación del aire, donde las ganacias ambientales obtenidas por la instalación de dispositivos descontaminantes en los vehículos o la utilización de combustible sin plomo pueden ser revertidas por un patrón de crecimiento urbano que provoca la extensión de los trayectos y tiempos de transporte, o por el incremento del congestionamiento vial.

Utilizando el marco analítico expuesto en el segundo punto, la estrategia ambiental debe contener propuestas de acción no sólo sobre aspectos del medio ambiente en sentido estricto (como la preservación de bienes ambientales o la restricción de su uso), sino también sobre la población, las actividades, el territorio y la gestión.

Así, por ejemplo, debe proponer acciones tendientes a la reducción de los niveles de pobreza, vulnerabilidad y exclusión social de la población que habita y utiliza la RMSS. Debe, en lo que se refiere a las actividades y al territorio, incluir, además de la regulación de las primeras, que sean permitidas la definición de los usos del suelo y sus restricciones, y las características de las redes de infraestructura, lo que remite al Plan de Desarrollo Metropolitano. En lo concerniente a la gestión, la estrategia debe proponer una modemización y descentralización de las inslancias responsables y la creación de mecanismos de participación ciudadana en esta problemática.

Consideramos que sin el establecimiento de estos lineamientos generales y su aplicación, son limitados los beneficios para definir y alcanzar metas de tipo cuantitativo, por ejemplo, determinado porcentaje en la reducción de la generación de desechos sólidos. Toda estrategia debe, evidentemente, conducir al establecimiento de planes de acción concretos, pues de lo contrario carecería de sentido. Sin embargo, y aunque es más dificil percibir su importancia y aplicación, estos lineamientos 
de política general son indispensables, tanto para evitar la degradación ambiental como para contribuir a entender que los ecosistemas son totalidades que deben ser construidas también conceptualmente (Harvey, 1993). Con el objetivo de avanzar en la primer tarea, a continuación se presentan dos matrices. En la primera se sintetizan los objetivos de la estrategia y, en el segundo, los instrumentos para su ejecución.

\section{Cuadro 3}

Estrategia ambiental: objetivos

\begin{tabular}{|c|c|c|c|c|c|}
\hline \multicolumn{6}{|c|}{ Componentes } \\
\hline & \multicolumn{2}{|c|}{ Capilal nalural } & \multirow[b]{2}{*}{$\begin{array}{l}\text { Capilal } \\
\text { humano }\end{array}$} & \multirow[b]{2}{*}{$\begin{array}{l}\text { Capital } \\
\text { social }\end{array}$} & \multirow[b]{2}{*}{$\begin{array}{l}\text { Capital } \\
\text { edificado }\end{array}$} \\
\hline & $\begin{array}{l}\text { Recursos } \\
\text { ambientales }\end{array}$ & $\begin{array}{l}\text { Servicios } \\
\text { ambientales }\end{array}$ & & & \\
\hline Población & $\begin{array}{l}\text { Adecuar el uso } \\
\text { y desarrollo de } \\
\text { los recursos al } \\
\text { crecimiento } \\
\text { poblacional } \\
\text { estimado }\end{array}$ & $\begin{array}{l}\text { Ampliar } \\
\text { cobertura de } \\
\text { los servicios } \\
\text { ambientales } \\
\text { y optimizar } \\
\text { su uso por } \\
\text { parte de la } \\
\text { población }\end{array}$ & $\begin{array}{l}\text { Promover la } \\
\text { cducación } \\
\text { ambiental } \\
\text { en todos los } \\
\text { niveles }\end{array}$ & $\begin{array}{l}\text { Desarrollar } \\
\text { asociaciones } \\
\text { y redes } \\
\text { ambientales } \\
\text { urbanas }\end{array}$ & $\begin{array}{l}\text { Introducir } \\
\text { una visión } \\
\text { ambiental en } \\
\text { relación con las } \\
\text { edificaciones }\end{array}$ \\
\hline $\begin{array}{l}\text { Actividades } \\
\text { produclivas } \\
\text { y } \\
\text { reproductivas }\end{array}$ & $\begin{array}{l}\text { Reducción en } \\
\text { los niveles de } \\
\text { consumo de } \\
\text { recursos } \\
\text { ambicnıles }\end{array}$ & $\begin{array}{l}\text { Opimizar el } \\
\text { uso de } \\
\text { servicios } \\
\text { ambientales } \\
\text { en las diversas } \\
\text { aetividades }\end{array}$ & $\begin{array}{l}\text { Introducir una } \\
\text { visión ambiental } \\
\text { en la ejecución } \\
\text { de las } \\
\text { actividades }\end{array}$ & $\begin{array}{l}\text { Estimular las } \\
\text { asociaciones } \\
\text { ambientales } \\
\text { a Iravés de las } \\
\text { actividades }\end{array}$ & $\begin{array}{l}\text { Uiilizar con } \\
\text { crilerios } \\
\text { ambientales } \\
\text { en el uso de } \\
\text { las edifica- } \\
\text { ciones }\end{array}$ \\
\hline Territorio & $\begin{array}{l}\text { Determinar y } \\
\text { vigilar la } \\
\text { capacidad de } \\
\text { carga y los } \\
\text { umbrales } \\
\text { criticos }\end{array}$ & $\begin{array}{l}\text { Delerminar } \\
\text { y vigilar } \\
\text { la capacidad } \\
\text { de la oferta } \\
\text { de los } \\
\text { servicios } \\
\text { ambientales } \\
\text { que brinda el } \\
\text { territorio }\end{array}$ & $\begin{array}{l}\text { Crear } \\
\text { conciencia de } \\
\text { la relación } \\
\text { ser humano- } \\
\text { territorio }\end{array}$ & $\begin{array}{l}\text { Promover la } \\
\text { identidad } \\
\text { territorial }\end{array}$ & $\begin{array}{l}\text { Analizar la } \\
\text { relación entre } \\
\text { el terriıorio } \\
\text { y las edifi- } \\
\text { caciones }\end{array}$ \\
\hline Gestion & $\begin{array}{l}\text { Crear un marco } \\
\text { legal y } \\
\text { regulatorio que } \\
\text { contribuya a la } \\
\text { sosienibilidad } \\
\text { construclivos }\end{array}$ & $\begin{array}{l}\text { Crear un } \\
\text { marco legal } \\
\text { y regulatorio } \\
\text { que conlribuya } \\
\text { a la sosieni- } \\
\text { constructivos }\end{array}$ & $\begin{array}{l}\text { Desarrollar } \\
\text { la "ciudadanla } \\
\text { ambienlal" } \\
\text { con sus derechos } \\
\text { y debcres } \\
\text { constructivos }\end{array}$ & $\begin{array}{l}\text { Introducir la } \\
\text { dimensión } \\
\text { ambiental en } \\
\text { las redes } \\
\text { sociales } \\
\text { constructivos }\end{array}$ & $\begin{array}{l}\text { Introducir } \\
\text { criterios } \\
\text { embienlales } \\
\text { en los } \\
\text { procesos } \\
\text { consiruclivos }\end{array}$ \\
\hline
\end{tabular}




\section{Cuadro 4}

Estrategia ambiental: instrumentos

\begin{tabular}{|c|c|c|c|c|}
\hline \multicolumn{5}{|c|}{ Procesos } \\
\hline & Planificación & Regulación & Administración & Inversión \\
\hline Población & $\begin{array}{l}\text { Mecanismos de } \\
\text { participación } \\
\text { ciudadana en los } \\
\text { procesos de } \\
\text { planificación } \\
\text { ambiental }\end{array}$ & $\begin{array}{l}\text { Instrumenlos } \\
\text { de vigilancia } \\
\text { ambiental } \\
\text { particlpativos }\end{array}$ & \multirow{2}{*}{$\begin{array}{l}\text { Mecanismos de } \\
\text { participación } \\
\text { ciudadana y } \\
\text { empresarial en } \\
\text { programas } \\
\text { ambientales }\end{array}$} & $\begin{array}{l}\text { Premios por } \\
\text { inversión } \\
\text { ambiental } \\
\text { ciudadana }\end{array}$ \\
\hline Actividades & $\begin{array}{l}\text { Planes } \\
\text { especificos por } \\
\text { sector según } \\
\text { niveles de uso } \\
\text { de recursos y } \\
\text { servicios } \\
\text { ambientales, y } \\
\text { niveles de } \\
\text { conlaminación } \\
\text { producida }\end{array}$ & $\begin{array}{l}\text { Planes de } \\
\text { aulomoniloreo }\end{array}$ & & $\begin{array}{l}\text { Incentivos por } \\
\text { inversiones en } \\
\text { prolecclón } \\
\text { ambiental y } \\
\text { descontaminación }\end{array}$ \\
\hline Territorio & \multirow[t]{2}{*}{$\begin{array}{l}\text { Planes de } \\
\text { ordenamiento } \\
\text { ambiental }\end{array}$} & \multirow[t]{2}{*}{$\begin{array}{l}\text { Marco regulatorio } \\
\text { ambiental }\end{array}$} & \multirow{2}{*}{$\begin{array}{l}\text { Creación de } \\
\text { institueiones } \\
\text { ambientales en } \\
\text { los distintos } \\
\text { niveles de } \\
\text { inlervención }\end{array}$} & \multirow{2}{*}{$\begin{array}{l}\text { Cartera de } \\
\text { proyectos } \\
\text { priorizados } \\
\text { concertadamenle } \\
\text { por los } \\
\text { principales } \\
\text { actores }\end{array}$} \\
\hline Geslión & & & & \\
\hline
\end{tabular}

Las propuestas contenidas en los cuadros anteriores revelan la extrema complejidad de la tarea de construir una estrategia ambiental para la RMSS, que contribuya a la sostenibilidad del país. Ella constituye, no obstante, un deber ineludible si se quiere combatir la tragedia que rodea a los bienes comunes, como son los recursos y servicios ambientales, que tienen su origen en el hecho de que los usuarios de los mismos buscan maximizar sus utilidad individual, lo que conduce, por último, a su destrucción por la sobreutilización a que son sometidos.

La sostenibilidad de El Salvador en el futuro depende de que se construya una estrategia ambiental para el conjunto del país, teniendo en cuenta ls vinculaciones que tiene con los países vecinos y las estrategias ambientales para ámbitos específicos. Uno de ellos es la RMSS por la importancia de la misma que hemos descrito anteriormente. 
La construcción de estas estrategias ambientales requieren de una adecuada combinación de los diferentes niveles institucionales: local, metropolitano, regional, nacional e internacional; de la transformación de los simples grupos de intereses en verdaderos actores sociales; de un justo equilibrio entre medio ambiente y desarrollo y, finalmente, de la construcción de una nueva forma de gestión ambiental, particularmente para el caso de la RMSS.

\section{Referencias bibliográficas}

Banco Mundial, Polticica urbana y desarrollo económico: un programa para el decenio 1990, Washington, 1991.

Bartone, Carl y cols., Toward Environmental Strategies for Cities, Urban Management Program, The World Bank, Washington, 1994.

Bommer, Julian; Salazar, Walter y Samayoa, Ricardo, Riesgo Sísmico en la Región Me. tropolitana de San Salvador, PRISMA, San Salvador, 1998.

Brundtland, Gro Harlem y cols., Our Common Future, Oxford University Press, Nueva York, 1987.

Burgess, Rod; Carmona, Marisa y Kolslee, Theo, The Challenge of Sustainable Cities, Zed Books, Londres, 1997.

Coleman, James, "Social Capital in the Creation of Human Capital", American Journal of Sociology, Eslados Unidos, 1988.

Cuervo, Luis Mauricio y cols., La Economía de la Región Metropolitana de San Salvador, PRISMA, San Salvador, 1998.

García, Gilberto, Contaminación por Desechos Sólidos en la Región Metropolitana de San Salvador, PRISMA, San Salvador, 1998.

Godelicr, Mauricc, L'idéel et le matériel, Fayard, París, 1984.

Gómez, Ileana, Procesos Ambientales y Actores en la Región Metropolitana de San Salvador, PRISMA, San Salvador, 1998.

Harvey, David, "The Nature of Environment: the Dialectics of Social and Environmental Changes", The Socialist Register, Londres, 1993.

Larios, Silvia, Transporte Urbano y Conıaminación del Aire en la Región Metropolitana de San Salvador, PRISMA, San Salvador, 1998.

Lungo, Mario, "Los Planes de Desarrollo Urbano. Construyendo nuevas relaciones de gobernabilidad urbana", en Gobernabilidad Urbana en Centroamérica, Mario Lungo (compilador), San José: FLACSO/GURI, Costa Rica, 1998.

Lungo, Mario, "Medio Ambiente y población en las ciudades centroamericanas", en Es. tudios Sociales Centroamericanos, No. 49, San José, Cosla Rica, 1989.

Lungo, Mario; Oporto, Francisco y Chinchilla, Roberto, La Evolución de la Red Urbana y el Desarrollo Sostenible en El Salvador, PRISMA, San Salvador, El Salvador, 1996.

Moser, Caroline, Confronting Crisis. A Comparative Study of Household Responses to Poverty and Vulnerability in Four Poor Urban Communities, The World Bank, Environmentally Sustainable Development Studies and Monographs Serics No. 8, Washington, 1996.

MSPYAS, "Salud Pública en Cifras", Anuario No. 27, San Salvador, El Salvador, 1995.

Orellana, Eliseo, Perfil Epidemiologico y Medio Ambiente en la Región Metropolitana de San Salvador, PRISMA, San Salvador, 1998. 
PRISMA, El Salvador: Dinámica de Degradación Ambiental, San Salvador, El Salvador, 1995.

Serageldin, Ismail, Sustainabillity and the Wealth of Nations: First Steps in an On going Journey, Preliminary Draft, Third Annual World Bank Conference on Environmental Sustainable Development, Washington, 1995.

Stedman, Pamela y Reed, David, Los retos del desarrollo sostenible y la reforma del Banco Mundial y del FMI, Boletín, PRISMA No. 15, San Salvador, 1996.

Stren, Richard; While, Rodncy y Whitney, Joseph, Sustainable Cities, Westview Press, Boulder, 1992.

UCA/FIAES, Investigación aplicada sobre el impacto ambiental de la contaminación del agua en las cuencas de los ríos Sucio, Acelhuate y Cuaya, Universidad Centroamericana José Simeón Cañas, San Salvador, enero, 1997.

UNDP, Cities, People and Poverty, Nueva York, 1991.

Viceministerio de Transporte, Plan Maeslro del Transporte Vehicular del Area Melropolitana de San Salvador, Ministerio de Obras Públicas, San Salvador, El Salvador, 1997.

VMVDU, Plan Maestro de Desarrollo Urbano del Arca Metropolitana Ampliada de San Salvador, Ministerio de Obras Públicas, San Salvador, 1997.Notas

\section{Notas}

1. Trabajo elaborado para la Universidad de Comell, julio de 1998.

2. Aunque importante, esta llamada de atención sobre la relación cntre el fenómeno urbano y el desarrollo sostenible no fue tomada con la debida importancia, tal como se manifestó en el lugar secundario que le fue otorgado cinco años más tarde en la Conferencia de Río de Janeiro.

3. Esta concepción se basa en que el ser humano ocupa el lugar central y en cl respeto a la capacidad de la naturaleza.

4. El Programa de Gestión Urbana es un esfuerzo conjunlo del PNUD, el Centro de las Naciones Unidas para los Asentamientos Humanos, HABITAT, y cl Banco Mundial.

5. El Area Metropolitana de San Salvador, la cual abarca 14 de los 24 municipios de la RMSS, aclualmente es la única árca consolidada a nivel de gestión, dada la existencia del Consejo de Alcaldes del Area Mctropolitana de San Salvador (COAMSS) y de la Oficina de Planificación de la misma (OPAMSS).

6. Entre 1985 y 1995 , las exportaciones tradicionales disminuyeron del 71 al 26 por ciento respeclo al lotal de exportaciones; micntras que las exportaciones no tradicionales y la maquila aumentaron del 28 al 35 por ciento y del 1 al 40 por ciento, respectivamente.

7. Ejemplo de lo anterior es la elaboración y comienzo de la implementación del PLAMADUR para el AMSS, y la próxima elaboración de un Plan de Desarrollo para el Valle de Zapotitán, incluido en nuestra definición de la RMSS. 